\title{
Formation Control of Swarm Robots Using Mobile Agents
}

\author{
Yasushi Kambayashi" \\ Department of Computer and Information Engineering \\ Nippon Institute of Technology, 4-1 Gakuendai \\ Miyashiro, Saitama 345-8501, Japan \\ yasushi@nit.ac.jp \\ Hideaki Yajima*, Tadashi Shyoji†, Ryotaro Oikawa \\ and Munehiro Takimoto§ \\ Department of Information Sciences \\ Tokyo University of Science \\ 2641 Yamazaki Noda, Chiba 278-8510, Japan \\ *6316638@ed.tus.ac.jp \\ †6318515@ed.tus.ac.jp \\ †oikawa@cs.is.noda.tus.ac.jp \\ §mune@is.noda.tus.ac.jp \\ Received 18 November 2018 \\ Revised 13 February 2019 \\ Accepted 5 March 2019 \\ Published 7 May 2019
}

\begin{abstract}
In this paper, we propose an algorithm for controlling a fleet of swarm robots that construct three-dimensional forms. The swarm robots coordinate with each other through network communication, and compose formations such as polyhedrons presented as spherical coordinates. Our control algorithm achieves communication through mobile software agents, which introduce control programs to robots that initially have no information about the formation. Mobile software agents are autonomous objects that can migrate from one robot to other robots through a communication network and can deliver control programs as they are needed. We have made our swarm robot system to mimic the behaviors of the leafcutter ants. A leafcutter ant is a typical social insect and uses pheromone for communication. In our robot control system, we have implemented ants and pheromones as mobile software agents. We call the mobile software agents that drive the mobile robots as ant agents, and call the other agents that provide communication as pheromone agents. The ant agents drive the swarm robots to locations identified by the pheromone agents. Each ant agent has only partial information. There is no need for either a central control or an agent that has the entire design of the formation. In order to diffuse the partial information among the neighboring robots, each ant agent generates pheromone agents and dispatches them to the surrounding robots. Dispatched pheromone agent
\end{abstract}

\footnotetext{
\ Corresponding author.
}

This is an Open Access article published by World Scientific Publishing Company. It is distributed under the terms of the Creative Commons Attribution 4.0 (CC-BY) License. Further distribution of this work is permitted, provided the original work is properly cited. 


\begin{abstract}
looks for a proper ant agent to influence towards a desired relative location. It is the ant agent that actually drives the robot by following the guidance of the pheromone agent, and the collective actions of ant agents and pheromone agents achieve the composition of the objective formation. We have implemented a simulator based on our algorithm and conducted numerical experiments. The results demonstrate that our mobile robot control system is feasible and efficient in practice in practical situations.
\end{abstract}

Keywords: Mobile agent; multi-agent system; multi-robot system.

\title{
1. Introduction
}

This paper is an extension of the work originally presented in SAI Intelligent Systems Conference 2017. ${ }^{1}$ The work presented our basic idea of multiple software mobile agents that mimic the behaviors of ants and influences of pheromones, and demonstrated that the idea is applicable to compose two-dimensional formations of multiple mobile robots. In this paper, we have extended the work to show that our system can direct mobile robots to compose three-dimensional polyhedrons. We report the results of numerical experiments and add extensive background researches.

The recent progress of hardware such as miniaturization and ever higher performance of computer devices has made robot systems rapidly advance not only in terms of the behavior of the robot but also in the method of controlling the robot. One such advanced control method is the employment of mobile software agents. A mobile software agent is an autonomous object that moves among a set of mobile robots and controls the mobile robot as hardware. In particular, a robot control system based on multiple agents has introduced modularity, reconfigurability and extensibility for multi-robot systems. ${ }^{2}$ Additionally, mobile software agents make robot control systems simple in distributed environments.

On the other hand, a system based on simple multi-agents is not ideal, because multi-agent systems may cause excessive interactions among agents and impose heavy communication loads in the system. Heavy communication loads make the multi-agent system less useful for controlling multiple robots, because a multi-robot system is unlikely to enjoy the luxury of a rich network environment with wide bandwidth. In order to mitigate the problems of excessive communication, we have investigated and devised mobile agent methodologies for distributed environments. In a mobile agent system, each agent can move autonomously from one computer to another. Since a mobile agent can bring the necessary functionalities with it and perform its tasks autonomously, it can reduce the necessity for interaction between computers. In a case where communication is minimally performed, the mobile agent needs to establish a connection between computers only when it performs migration. A mobile agent system is particularly effective in an ad hoc network environment where connections can be intermittently established. ${ }^{3}$

The migration property of mobile agents contributes to the flexibility and efficiency in the use of the robot resources in a multiple-robot system. A mobile agent can migrate to the robot that is most conveniently located for a given task. Since agent migration is much easier than physical motion of a robot, agent migration also 
contributes to reducing power consumption. ${ }^{4}$ In an environment where several different tasks must be achieved simultaneously, we can coordinate the control of a large number of heterogeneous robots with multiple mobile software agents. We can employ a policy of choosing idle robots most suitably equipped and most conveniently located to undertake the task. Thus we achieve the most efficient use of robot resources. We can also enjoy the energy saving effect.

In this paper, we focus our attention on the formation control of mobile robots. We propose an algorithm for controlling a fleet of actual swarm robots that are able to self-organize into specified formations. This is one of the most important capabilities in order to complete tasks that require collective action. The ability to compose formations is especially important when individual robots have limited capabilities. For example, robots may aggregate for the coordinated activity of search and rescue in the event of a disaster, collectively transporting a large object, pioneering and mapping unknown areas or maintaining a formation for the construction of buildings. ${ }^{5}$ We introduce a novel control and management system for rapid organization of mobile robots into a desired formation, more efficient use of network communication capacity and reduced energy consumption by the physical components of this process. In our system the complete design is known to the external users' control system which releases the correct mobile software agents into the environment to create the formation desired.

In most other formation control algorithms, each robot requires the information about the entire formation, which is unlikely for real-world applications. In our algorithm, on the other hand, each robot requires only the local neighbor information. An iterative process refines the relative locations of the members of the group to produce the final structure with the precise shape and dimensions specified by the user to the external user machine. The swarm robots in our system coordinate with each other by using network communication, and compose formations. The formations include specific polyhedrons that are represented using spherical coordinates. Our control algorithm communicates via the network to the robots using mobile software agents. Therefore, we can introduce control programs to robots that initially have no information about the formation. Since the mobile agents can migrate from one robot to other robots through the communication network, they can bring control programs as they are needed. As a result, we can introduce new control programs to the fleet of mobile robots from outside as they need them as tasks are completed or are modified.

We have made our swarm robot system to mimic the behaviors of the leafcutter ants. A leafcutter ant is a typical social insect and uses pheromone as a communication means. Even though we have pursued the idea of ant colony optimization (ACO), the implementation is drastically different from the traditional ACO. We have implemented ants and pheromones in ACO as actual mobile software agents, and the cooperation of these two kinds of mobile agents controls the mobile robots to compose a formation. ${ }^{6-8}$ The ant agents (AAs) drive the mobile robots and attract each other using pheromone agents (PAs). Thus the ant agents eventually drive the 
mobile robots to appropriate locations in order to achieve the composition of the target formation.

To operate in our system, the ant agents need only the local information which consists of their positions relative to nearby robots. Each ant agent is designed to drive a robot to a specific position relative to its neighbors. Therefore, an ant agent first looks for arbitrary idle robot, identifies its neighbors' locations and identities and then the ant agent migrates to another robot that is more conveniently located relative to other robots. When it finds a convenient robot, it then starts driving it to the relative position using the given relative coordinates for that ant agent. Thus, the behaviors of mobile agents and robots driven by them enable a robot close to each part of a target formation to occupy that location, contributing to efficient use of idle robots, efficient convergence and reduced energy consumption.

Our system consists of robots and two kinds of mobile software agents. We assume that the robots have simple capabilities of movement such as rolling wheels, measuring distance and angle through an optical camera and other sensors. In addition to mobile capability, the robots have some communication devices with which they can communicate with each other through a communication network.

All the controls for the mobile robots are achieved through the mobile agents. These are ant agents and pheromone agents. In our algorithm, ant agents attract each other to suitable positions as shown in Fig. 1. They autonomously adjust their relative positions without any central coordination. In this figure, robots are represented as circles, and the desired neighbor locations of each robot are represented as stars to which vectors relative to the robot point. The vectors are represented as black arrows that indicate the distance and direction of the locus that the originating robot needs to see occupied by a neighbor in order to compose the formation. Colored arrows represent the direction of motion that the second robot computes and moves in response to pheromone agent sent by the first robot. Successive iterations of determination of position and release of pheromone by the neighbors result in the movement of the two robots into the appropriate relationship. We have implemented a simulator based on this algorithm and conducted numerical experiments to demonstrate that our mobile robot control system is feasible in practice in the real world.

The structure of the balance of this paper is as follows. In Sec. 2, we describe the background. Section 3 describes the method for composing a formation using mobile

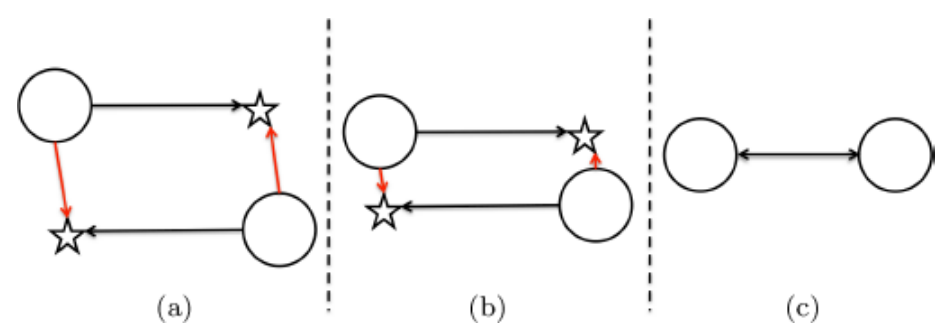

Fig. 1. The process of formation. 
agents. Our mobile agent system consists of two kinds of mobile software agents, namely ant agents and pheromone agents. We describe each of them and present the role and algorithm to implement the robot formation control system. In Sec. 4, we report the results of numerical experiments using a simulator based on our algorithm. We describe an environment constructed according to data actually obtained with real equipment. Finally, we conclude our discussions in Sec. 5.

\section{Background}

We have previously presented a novel framework for controlling intelligent multiple robots connected by wireless communication networks. ${ }^{2,4}$ This framework helps users to construct intelligent robot control software by migration of mobile agents. We have developed a novel method to control coordinated systems using mobile agents. Since the system can be configured dynamically through migration of agents, the control software can be dynamically assembled while they are running. Dynamically extending control software by the migration of mobile agents enables us to make the base control software relatively simple, and to add functionalities one by one as we learn about the working environment. Thus we do not have to give the intelligent robot complete instructions from the beginning or make the robot learn by itself. In this approach, we can send intelligence, i.e. additional instructions and new data, later via new agents. We have implemented a team of cooperative search robots. ${ }^{2,4,6-9}$ We have successfully shown that our framework for controlling multiple robots is not only feasible, but also effective enough for practical use. In addition, we have found that our framework contributes to energy saving in the operation of multiple robots. ${ }^{4,9}$ Our approach has provided significant energy saving for search robot applications. This feature is extremely important when implementing our multi-robot system using battery-driven robots.

In recent years, ACO has been gradually established as a popular algorithm. ${ }^{10,11}$ ACO was an algorithm inspired by the behaviors of social insects such as ants. In the algorithm, agents that mimic the behaviors of ants communicate with each other by an indirect communication mediated by modifications of the environment called stigmergy. The behavioral scientists Goss et al. found that ants exchanged information by laying down a trail of a chemical substance called pheromone that is followed by other ants. ${ }^{12,13}$ In order to solve the traveling salesman problem, Dorigo and Gambardella developed an algorithm called Ant Colony System (ACS) which is based on ACO. ${ }^{11} \mathrm{ACO}$ is used to solve various optimization problems, and many derivative algorithms are also proposed. Today, they are considered as one of the major research themes. Deneubourg et al. studied the ants' behaviors about corps gathering and brood sorting, and proposed ant-like robots that simulate ant corps gathering and brood sorting behaviors. ${ }^{14}$ Wang and Zhang have improved Deneubourg et al.'s biologically-inspired behavioral algorithm and have proposed multi-robot system that performs collective sorting. ${ }^{15}$ 
Lumer and Faiesta have further improved Deneubourg et al.'s model and proposed a new algorithm that is called Ant Colony Clustering (ACC).${ }^{16}$ In their paper, they introduced a new method for finding structures in complex datasets, and successively classifying data into a few clusters. Mizutani et al. have proposed to implement pheromones as mobile software agents, and demonstrated that it is feasible to construct multi-robot systems using mobile software agents as ants and pheromones for implementing ACC. ${ }^{6}$ In their algorithm, they have introduced two kinds of mobile software agents, i.e. ant agents and pheromone agents. The ant agents represent artificial ants that manage search carts as objects and drive them to the quasi-optimal positions. ${ }^{17}$ The pheromone agents are generated by the ant agents when their carts reach a cluster, and repeatedly migrate to other carts, and guide ant agents to drive their carts to the same cluster. The pheromone agents act as a beacon for attracting ant agents, and thus collecting carts into a few clusters. We have successfully instructed a group of robot carts to self-organize into multiple lines. ${ }^{18-20}$

Oikawa et al. have taken advantages of the pheromone communication of ants and proposed a multi-robot system to compose formations. ${ }^{21-23}$ In their method as well as Mizutani and Shintani's proposals, they have implemented ants and pheromones as mobile software agents and demonstrated that it is feasible to use them to compose two-dimensional formations. Ant agents generate pheromone agents that have partial information about the formation and dispatch them to other ant agents. When the pheromone agents arrive to ant agents, they start to guide the ant agents to the appropriate locations. Once pheromone agents are generated, pheromone agents migrate to other robots to find the target ant agents, because the target ant agents may be far from the ant agents from which they originated. In such a case, the pheromone agents have to hop the robots many times to find the target ant agents. When ant agents receive the pheromone agents, the ant agents drive robots to the locations indicated by the pheromone agents. They also proposed a control algorithm for composing formations of swarm robots based on their distributed system using many mobile software agents. ${ }^{21-23}$ For this approach, they introduced two kinds of mobile software agents, guide agents and node agents. The guide agent traverses all the robots and calculates the conceptual barycenter of the robots, and then calculates the suitable locations of the formation using the barycenter and generates node agents that drive robots to the calculated locations. Each node agent migrates to the robot that is nearest to the target location, and drives that robot to the location. In other words, the guide agent must act as the central coordinator.

The most important and interesting projects in the multi-robot system world are the SWARM-BOTS project and its successor Swarmanoid project led by Dorigo. ${ }^{24-26}$ They have successfully developed a new robotic system based on swarm robotics technologies. We have learned a great many concepts as well as techniques from the project. A swarm robotics system is composed of many small, simple, mobile robots called swarm robots. They are tightly interacting and cooperate through indirect communication with each other to achieve common goals. Many of the swarm robotics projects are based on the theoretical studies of social insects such as ants, 
and the SWARM-BOTS project is not an exception. Like our project, many of the developments and experiments carried out within the project are directly inspired by the collective behaviors of ants. ACO and ACC are intensively employed for their robot control algorithms. Their study especially focuses on the mechanism that governs the processes of self-organization and self-assembly of multiple swarm robots as artificial autonomous agents. ${ }^{27,28}$ For example, their swarm robots can cooperatively retrieve and transport a heavy object. ${ }^{29,30}$ In addition to the cooperative transportation, the swarm robots utilize physical links to serve as support when they have to pass over a gap wider than a single robot or when they have to pass through a steep convex and concave region. ${ }^{31}$

In SWARM-BOTS project, Dorigo and co-workers extensively use the artificial neural networks to shape their evolutionary algorithms. ${ }^{32}$ They claim that the solutions found by evolution are simple, and the evolutionary algorithms can be generalized to various environments. They have demonstrated that evolutionary methods are able to produce self-organized systems that rely on simple and general rules. On the other hand, unlike the SWARM-BOTS project, we have developed a swarm robotics system that extensively uses mobile software agents. Mobile software agents are independent executable programs that autonomously migrate from a computer to another computer while executing certain tasks. As we demonstrate in this paper, we can introduce new functionalities as new control software from outside of the multi-robot system through mobile software agents.

As the other related field, we have considered the problem of optimal multi-robot path planning (MPP) on graphs. ${ }^{33,34}$ MPP has been studied since 1980s and found hard to solve. ${ }^{35}$ The reason why the problem is difficult is that planning collisionfree paths for multiple robots leads to an enormous state space due to the great amount of coupling between the robots' paths, and it is shown that finding the optimal solution is NP-hard. ${ }^{36}$ The core of this intractability is how to avoid collisions of mobile robots. Formation control has the same type of problem. When a fleet of robots try to compose a dense formation, they cannot avoid collision. When they try to avoid each other, the evasion activities cause chaotic situations. We have dramatically solved this problem through the exchange of the agents that drive colliding robots so that the robots keep moving by simply changing to the directions of their new agents. This solution demonstrates yet another useful aspect of mobile software agents.

In the formation research area, several kinds of swarm controlling methods have been proposed. Balch and Arkin proposed behavior-based motor scheme control. ${ }^{37}$ They have devised a control method called behavior-based strategy that defines simple behaviors or motion primitives for each robot. Lewis and Tan proposed one of the pioneering works of virtual-structure strategy. ${ }^{38}$ The virtual-structure strategy considers the entire formation as a rigid body, and the motion of each robot is translated from the motion of the virtual structure. The motion is determined by the dynamics of virtual structure. Das et al. proposed a control technique called leaderfollowing strategy. ${ }^{39}$ This strategy uses a feedback linearization control method, and 
it is one of the most popular control techniques. In the leader-following strategy, some robots are selected as leaders, and the other robots follow the leaders.

Some methods have a limited class of shapes that they can form. Unsal and Bay proposed a formation method that forms shapes of rings and circles. ${ }^{40}$ In their approach, robots issue beacons and instruct other robots to remain in their position and keep a certain distance. Mamei et al. proposed a similar method that composes a crude polygon and uses message hop count instead of a proximity sensor. ${ }^{41}$ Kondacs proposed an approach that can form many kinds of shapes. The approach is inspired by biological agents that grow and die. ${ }^{42}$ The notable characteristic of this approach is the ability to self-repair. The approach uses global and local compilations to automatically generate an agent.

Stoy and Nagpal have proposed one of the most closely related approaches to ours. They have successfully constructed multi-robot systems that compose threedimensional formations by using self-assembly and self-reconfigurable modular robots. ${ }^{43}$ Although this system generates a wide variety of formations, it cannot create solid shapes because agents may block each other and create internal holes that agents cannot reach. In order to avoid this problem, they focus on scaffolds and porous shapes. Gordon et al. proposed a method that forms arbitrary shapes sharing common coordinate systems. ${ }^{44}$ Their method, however, involves significant central computation. Although Rubenstein and Shen proposed a method that can self-repair, their method can form only polar shapes. ${ }^{45}$

Cheng et al. proposed a formation generation algorithm using a contained gas model. ${ }^{5}$ In their algorithm, robots act like particles in a container. Their method can construct almost arbitrary shapes, but it cannot compose a formation that does not have volume inside the shapes, such as a line formation. Moreover, although it can keep suitable distance between robots, the density of the robots inside the formation cannot be controlled. The algorithm cannot create a formation with different densities in the same formation.

In our formation approach, mobile agents are the main actors to compose a formation, and robots are just physical vehicles maneuvered by the mobile agents. The robots are only the means whereby mobile agents move to the target locations in the formation. Therefore, any traditional approach can be implemented on our mobile agent framework and the effectiveness in terms of its improved efficiency and energy savings can be obtained. Furthermore, a mobile agent has no volume, so that mobile agents do not block any other mobile agents. Thus, our approach can form not only line drawings but also arbitrary solid shapes and even formations with vacant areas inside.

\section{Composing Formations Using Mobile Agents}

In this study, we assume that the robots can physically move to any coordinates in three-dimensional space. Each robot can measure the distance and angle with an optical camera or other sensors, and communicate with others through wireless 
network such as Wi-Fi, ultrasonic or optical communication devices. Possible applications include aerial drones or autonomous underwater vehicles (AUVs) as the swarm robots.

\subsection{Basic idea}

Our system consists of robots and two kinds of mobile software agents. All the controls for the mobile robots are achieved through these mobile agents called ant agents. Each ant agent takes charge of one position in a formation and drives a robot to the position. We assume that all the ant agents are sent from the outside user machine to one of the reachable robots. Each ant agent does not know its own absolute position but has partial information of the formation consisting of its desired position relative to its neighbors in the completed form. For example, in a twodimensional environment, when the target formation is a circle and an ant agent currently resides on the robot $A$, and the robot $A$ 's neighbors are $B$ and $C$ as shown in Fig. 2, the ant agent on the robot $A$ has only the information of $B$ and $C$ represented as colored arrows in the figure $\left(\boldsymbol{P}_{A B}\right.$ and $\left.\boldsymbol{P}_{A C}\right)$.

Since ant agents carry vector values, we made an assumption that each robot orients itself by using a compass. Each robot can measure an angle with another robot based on that common orientation. Even though our robot system can be generalized by considering each robot's direction and adjusting them, this assumption makes our multi-robot system simple without losing much generality. When an agent on robot $A$ migrates to robot $B$, robot $B$ has a different coordinate (direction) from $A$ 's as shown in Fig. 3. As shown in the figure, $\theta_{A}$ is the angle to robot $B$ from robot $A$ and $\theta_{B}$ is to robot $A$ from robot $B$ and dotted arrows represent robot's angles. When the agent on robot $A$ migrates to robot $B$, the agent must translate the vector value it has such as the target location vector to adjust to

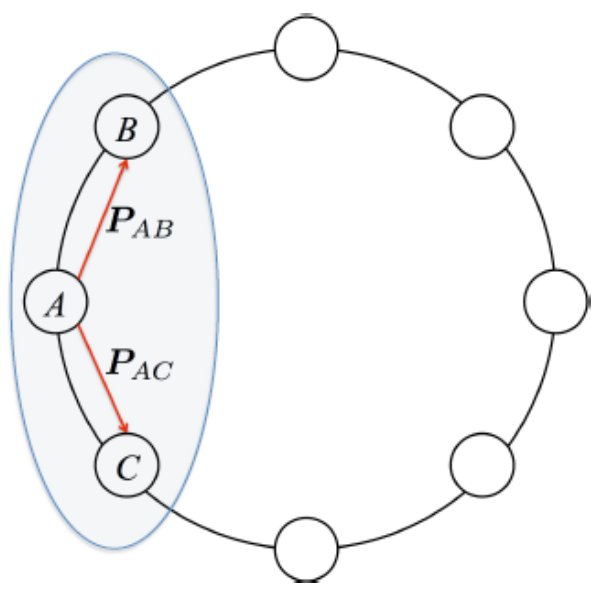

Fig. 2. Local shape information of the ant agent on $A$. 


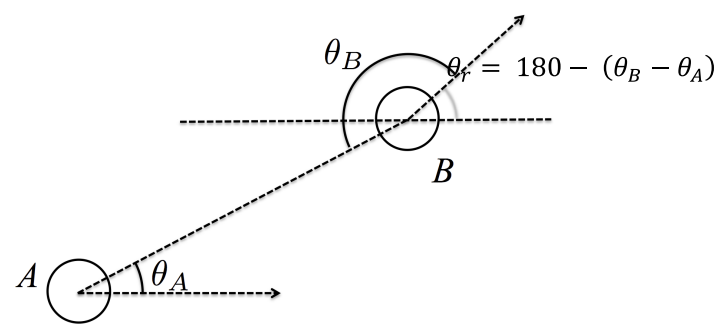

Fig. 3. Rotation angle.

$B$ 's coordinate. To do this, we calculate the difference $\theta_{r}$ of angles between the two robots as follows:

$$
\theta_{r}=180-\left(\theta_{B}-\theta_{A}\right) .
$$

In the algorithm that all the agents follow, if an ant agent cannot keep driving its robot due to some other robot that blocks its way, the ant agent migrates to the blocking robot and makes the ant agent on the blocking robot migrate back to the blocked robot so that the two driving ant agents exchange their robots. After exchanging robots, both ant agents resume driving their new robots to the ant agents' original target positions. This exchange functionality makes formation efficient, and particularly so for the formation of three-dimensional solid polyhedrons. Also, in the algorithm, each agent does not know the absolute coordinates of its own expected location. Instead, it only knows the relative coordinates of its neighboring ant agents, and it attracts them to the necessary relative coordinates using pheromone agents. A pheromone agent repeatedly migrates between robots while adjusting the target coordinates. Once it finds a specific ant agent whose position needs to be adjusted, it guides the ant agent to the target coordinate. Movement of a specific robot causes its neighbors to also need adjustment so the interaction of pheromone and ant agents iterates to attract neighbors to proper relative coordinates with respect to each other, enabling a shape to be composed without absolute coordinates.

\subsection{Ant agents and pheromone agents}

Our approach includes all the controls for the mobile robots into two kinds of mobile software agents, AAs and PAs. Each AA drives a robot according to the guidance of the PAs, while generating PAs to attract other AAs to drive their robots to occupy the neighboring positions of the robot that the AA wants. Since each AA needs the guidance of the PAs, AA must receive at least one PA before starting to drive its robot. The PA has vector information (given by AA that obtains through the robot's sensor system) pointing to neighboring positions of the robot the AA drives, traverses robots through communication network while generating its own clones to search for another AA to guide. The PA guides the AA based on its vector information, once it migrates to the robot that the AA drives. 


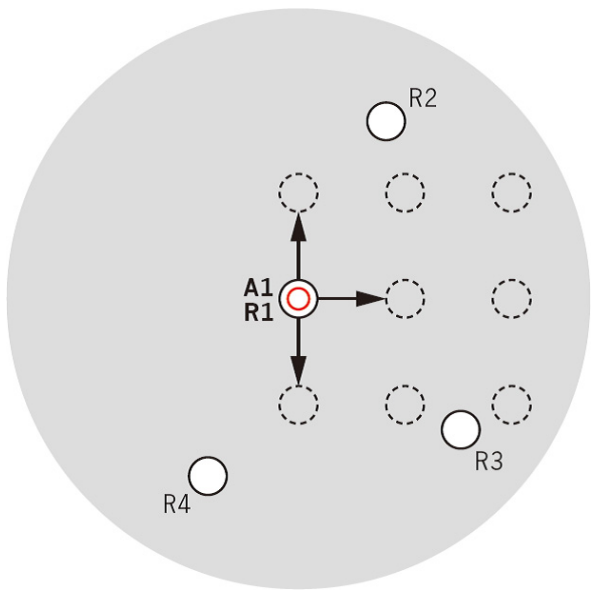

Fig. 4. Vectors of local information for a formation on the ant agent A1.

Figure 4 shows the objective formation and the vector values of local information that the ant agent $\mathrm{A} 1$ on robot $\mathrm{R} 1$ has. The objective formation is a solid square consisted of nine robots. They are represented as dotted circles, and R1 occupies the left center position. The ant agent A1 drives R1. R1's communication range is depicted by light gray circle. R2, R3 and R4 are in the circle, i.e. R1 can communicate with them. Therefore, A1 on R1 can generate and dispatch PAs to them. In our approach, each AA requires only local information about the objective formation. Local information is a set of vector values that represents the positional relationships between the imaginary neighbor AAs that compose the formation, i.e. the position the other AA should drive a robot and make it occupy. In the case of Fig. 4, the ant agent $\mathrm{A} 1$ on the robot $\mathrm{R} 1$ wants the robot $\mathrm{R} 2$ to come to its north position, the robot R3 to come to its east position and the robot R4 to come to its south position, respectively. The three vectors, which the ant agent A1 has, represent A1's desire. Therefore, A1 generates pheromone agents that have those three vector values to attract the ant agents A2, A3 and A4 with the robots R2, R3 and R4 to the desired positions. We assume the ant agents $\mathrm{A} 2, \mathrm{~A} 3$ and $\mathrm{A} 4$ drive the robots $\mathrm{R} 2, \mathrm{R} 3$ and $\mathrm{R} 4$, respectively.

Figure 5 shows the situation where $\mathrm{A} 1$ generates a pheromone agent $\mathrm{PA} 1$ with the vector that points to the desired location for the robot R2, PA1 migrates to the robot R2 and PA1 starts to guide A2 to the desired location. Since A2 has a vector pointing to $\mathrm{R} 1$, when PA1 arrives to R2, PA1 obtains the vector value, which is shown as the green arrow, PA1 synthesizes that vector with the original vector that PA1 carries, which is shown as the blue arrow in order to generate the vector that indicates R2's moving direction (the red arrow). For simplicity, A1 makes R1 to stay and only R2 to move, and A2 does not generate pheromone agent to attract R1. In the same manner, A1 generates and dispatches the pheromone agent PA2 to attract the robot R3 through guiding the ant agent A3 and generates and dispatches PA3 to attract R4 


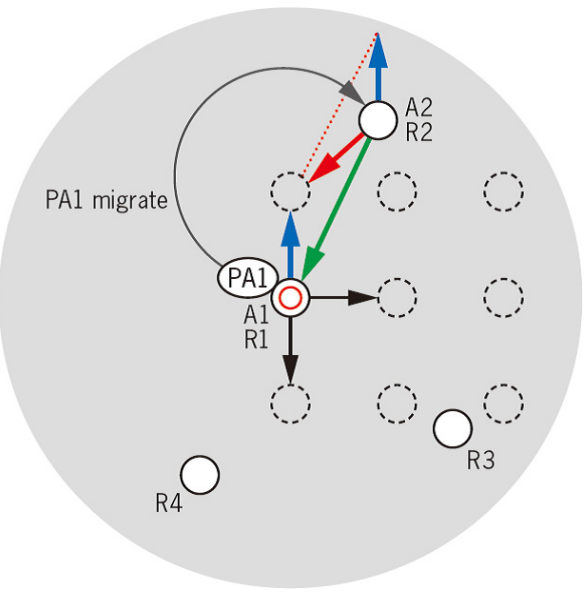

Fig. 5. A1 generates a pheromone agent and attracts A2 with its robot R2.

through guiding A4, respectively, as shown in Figs. 6 and 7. After those movements, the robots R2, R3 and R4 occupy the locations that the robot R1 wants and achieve a partial formation of square as shown in Fig. 8.

The movements of R2, R3 and R4 shown in Figs. 5-7 are too simplified, and do not reflect the real movements for composing a formation. In the figures, only the ant agent $\mathrm{A} 1$ on the robot $\mathrm{R} 1$ generates and dispatches pheromone agents, and the ant agents on R2, R3 and R4 do not generate pheromone agents. Therefore, they obediently follow the directions given by the ant agent on $\mathrm{R} 1$ through the pheromone agents they receive, i.e. PA1, PA2 and PA3, which are generated and dispatched by the ant agent $\mathrm{A} 1$ on $\mathrm{R} 1$. The ant agents on R2, R3 and R4, however, also generate pheromone agents and try to attract other robots to their own desired locations in a

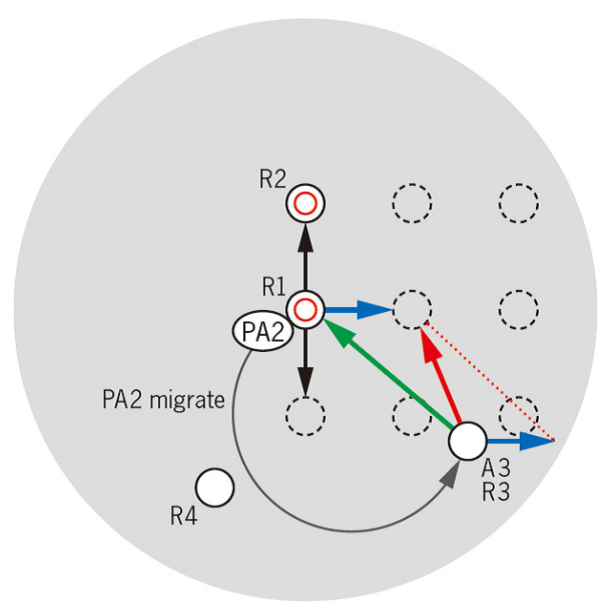

Fig. 6. A1 generates a pheromone agent and attracts A3 with its robot R3. 


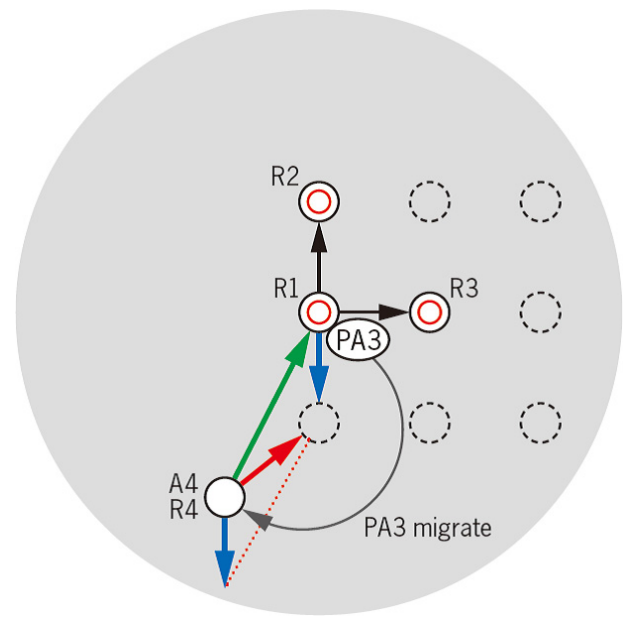

Fig. 7. A1 generates a pheromone agent and attracts A4 with its robot R4.

real situation. The four robots move simultaneously while they are also attracting and being attracted simultaneously.

The ant agents periodically generate pheromone agents while they are driving their robots. As a robot moves, the vector value that points to the robot that generates the pheromone agent, indicated in green arrows in the figures, varies. Even the vector value that indicates the desired location for neighboring robot may change. Therefore, every time when the driving ant agents receive pheromone agents with updated vector values, they have to re-calculate the vector values that indicate the driving directions, indicated in red arrows in the Figs. 5-7.

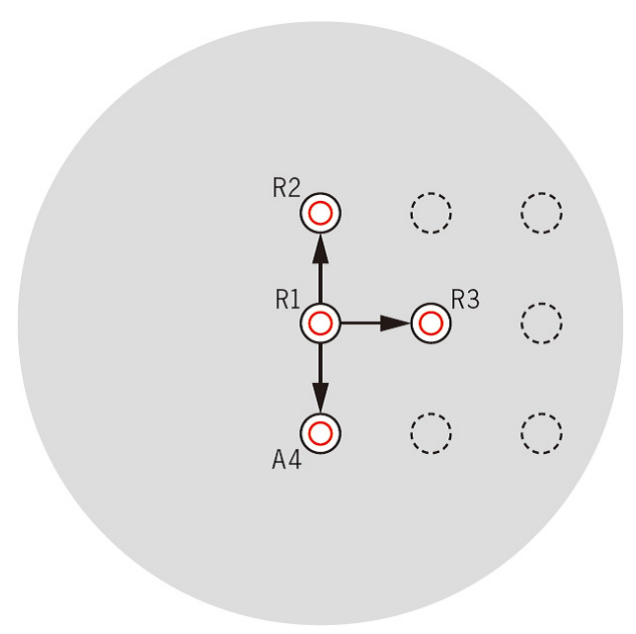

Fig. 8. A1 on R1 generated pheromone agents and accomplished to form a partial square by attracting A2, A3 and A4 with their robots R2, R3 and R4. 


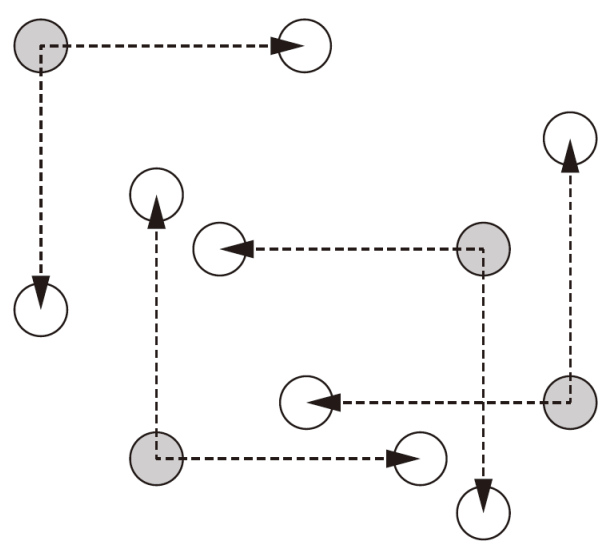

Fig. 9. Initial positions of ant agents in the field with vectors indicate the imaginary positions where they want their neighbors to occupy.

For example, let us consider four robots trying to compose a square formation. Since each robot has two neighboring robots, each ant agent that drives the robot has two vector locations that it wants other robots to occupy as shown in Fig. 9. The process in which the AAs compose the formation in cooperation with PAs is as follows.

First, four AAs traverse among the set of robots to find idle robots in the field, because some of the robots in the set may be already engaged in some other tasks. Once they find idle robots, they occupy a robot to drive. One AA occupies one robot. One AA has one or more vectors that point to imaginary neighbors. In this case, each AA has two vectors as shown in Fig. 9. Dark colored circles represent robots, and white circles represent the imaginary neighbors, i.e. the positions each robot wants other two robots to come and occupy. Once AAs recognize other robots through optical cameras, they start to generate PAs with vector values to each corresponding neighboring positions. Figure 9 shows four robots with AAs and two vectors for each robot to point to their ideal neighboring positions that they want other robots to occupy. The vector values from AA to the neighboring position are shown as arrows.

Second, PAs with the vector values repeatedly migrate to other robots while generating their clones in order to reach the robot with the AA that they want to guide. Figure 10 shows the situation where PAs have reached their target AAs. Since each AA has two vector values that points the ideal positions that it wants other robots to occupy, each AA produces two PAs to attract two robots as neighbors. Figure 10 shows the situation where each AA has just received two PAs from the neighboring AA. Red arrows show vector values that PAs hold. In the figure, the PA coming from upper left robot and arriving to the lower left robot is emphasized. As explained in Figs. 4-8, when the PA from upper left arrives to the lower left robot, it synthesizes its original vector (blue arrow) and the vector points to the original robot 


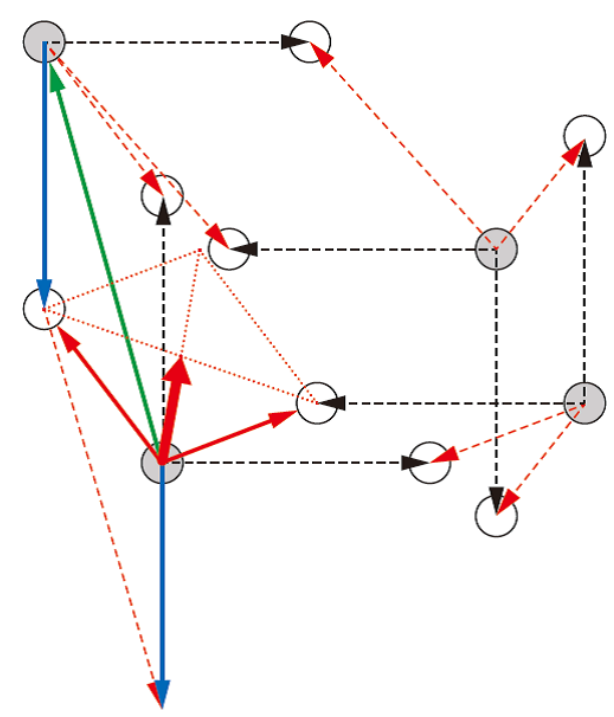

Fig. 10. Pheromone agents attract the target ant agent.

(green arrow) and produces the directional vector (solid red arrow). The robot, i.e. lower left one, moves along this vector (solid red arrow) until the next pheromone agent arrives. When a new pheromone agent arrives and the pheromone agent synthesizes the vector values and produces new directional vector, the AA synthesizes the two vectors and makes the robot move along that synthesized vector. For example, a pheromone agent from lower right robot arrives to this robot, this robot moves along the vector depicted by thick red arrow.

Thus each robot moves little by little as a pheromone agent arrives and AA on that robot synthesizes it into a guide vector. Finally, a robot with an AA settles to its own position that satisfies the positional relationship between the neighboring robots, so that an objective shape is composed as a result of the formation as shown in Fig. 11. Where and in what orientation the shape is composed in the field depend on the initial positions of robots because they move through attracting each other with PAs.

\subsection{Ant agents' behavior}

In this subsection, we describe the algorithm for AAs (Algorithm 1). AAs occupy idle robots in the field and drive these robots according to the guidance they receive from PAs. AAs repeatedly migrate via communication network through several robots while searching for an idle robot. Once the AA finds an idle robot, it occupies that robot, obtains location of its neighbors from the robot's sensors and generates PAs with vector values pointing to imaginary neighboring positions that the AA wants other robots to occupy. After that, the PAs diffuse themselves through migrations to find AAs to guide. An AA that receives a PA drives the robot on which it resides to 


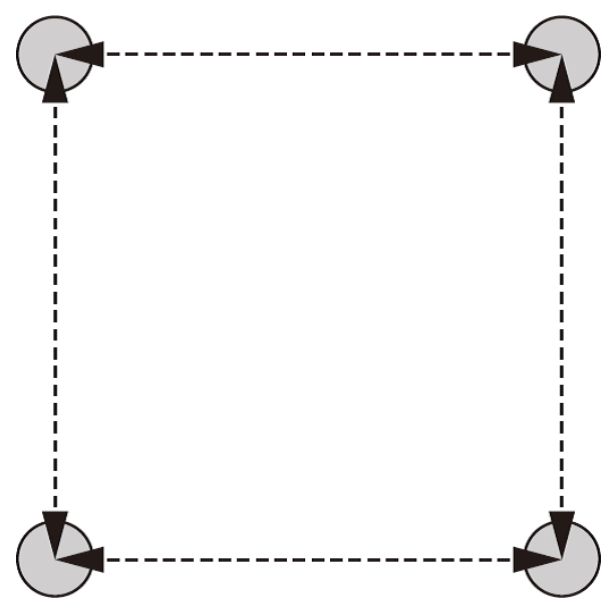

Fig. 11. All ant agents settled in the positions.

the target position according to the guidance of $\mathrm{PA}$. If an AA receives $n$ different $\mathrm{PAs}$ with vector value $V_{i}$ for the AA, these PAs are composed into a single PA with a vector value $V_{t}$ as follows:

$$
V_{t}=\frac{1}{n} \sum_{i=1}^{n} V_{i} .
$$

This causes the receiving AA to drive its robot to a position that is more optimal relative to the AAs that sent their PAs but not necessarily the optimum for any one of them. This begins the iterative process of moving all of the robots towards their final desired relative positions in space step by step.

In the process where AA approaches its own target position according to the guidance of $\mathrm{PA}(\mathrm{s})$, it does not drive its robot blindly. If the robot with the AA is far from the target position, the AA stops driving the current robot and migrates to another robot in the direction of the target position. This behavior contributes to efficient movement to the target position and avoids inefficient energy consumption

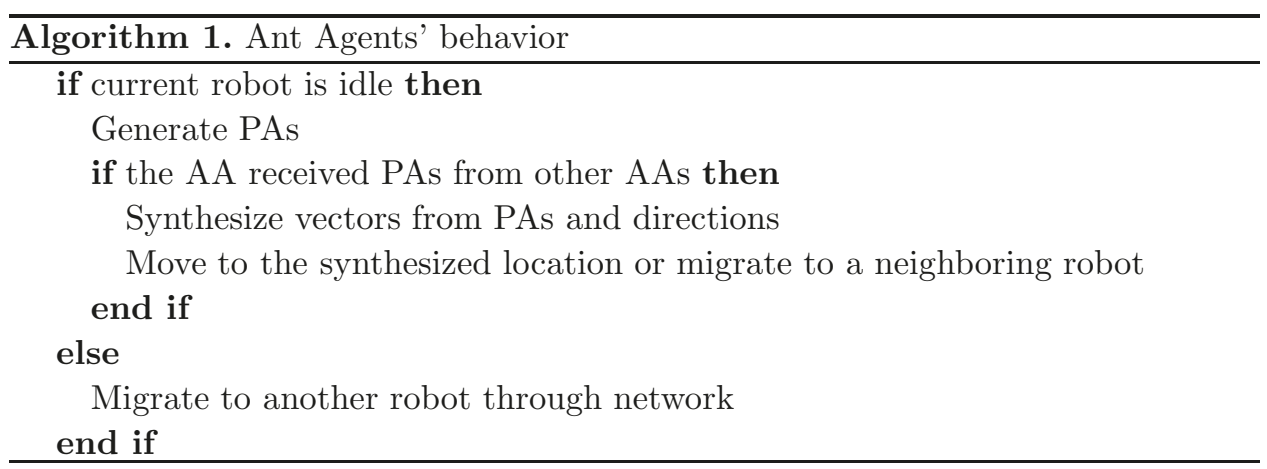


because driving a robot requires much more energy cost than driving an agent. In a situation where the robot is close to the target, however, the migration to another robot may cause the AA to pass through the target position, i.e. migrates to wrong (farther) robot. Thus, AA judges whether to migrate to the other robot or drive the current robot by comparing $V_{t}$ with the vector value from the other robot to the target position, $V_{\text {new }}$. The vector value is calculated as follows:

$$
V_{\text {new }}=V_{t}-V_{\text {another }} \text {. }
$$

As shown by Eq. (3), $V_{\text {new }}$ can be calculated by $V_{t}$ and the vector value from the current robot to the other robot, $V_{\text {another. }}$. If $\left|V_{\text {new }}\right|$ is less than a half of $\left|V_{t}\right|$, then AA migrates to the other robot; otherwise it drives the current robot along $V_{t}$.

Furthermore, we can assume the migration property for a situation when two or more robots collide. Let us consider a situation where two robots driven by two ant agents A1 and A2 collide. Since each robot has its own volume, progress of one robot blocks the progress of the other robot. Collision avoidance is known as an expensive maneuver in such a situation. At this time, in our agent-based system, A1 and A2 simply exchange their driving robots through reciprocal migrations. After that mutual transfer, both AAs keep their new robots moving according to the guidance of their PAs. Although the two physical robots leave in reverse directions, from the ant agents' point of view, they are just continuing to move straight to the original target positions. This exchange of robots enables AAs to proceed without complex maneuvering after a collision. This feature contributes to efficient convergence and reduction of energy consumption.

The process in which AAs exchange robots is as follows. Figure 12 shows that ant agents A1 and A2 are driving their robots according to the guidance of their respective PAs. Arrows in the figure are vector values that PAs carry for guiding A1 and A2. As we can infer from the figure, the two robots will collide as shown in Fig. 13 if they simply follow the guidance of the PAs. If, instead, A1 migrates to the robot that is driven by $\mathrm{A} 2$, and A2 migrates back to the robot driven by A1, so that they exchange the robots as depicted in Fig. 14, then the robots that were driven by A1 and A2 avoid expensive maneuvers to detour physically. This exchange of robots

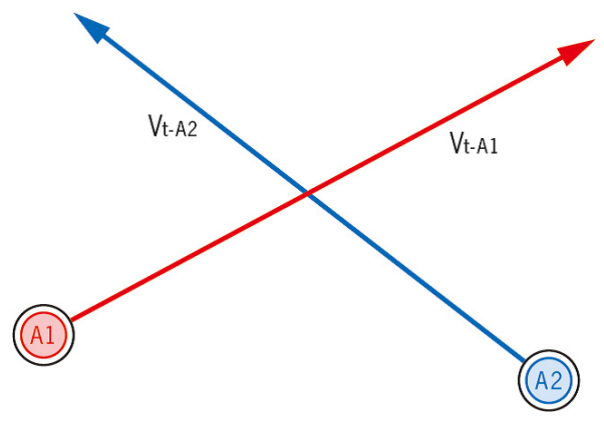

Fig. 12. AAs' original guidance to drive robots to the target positions. 


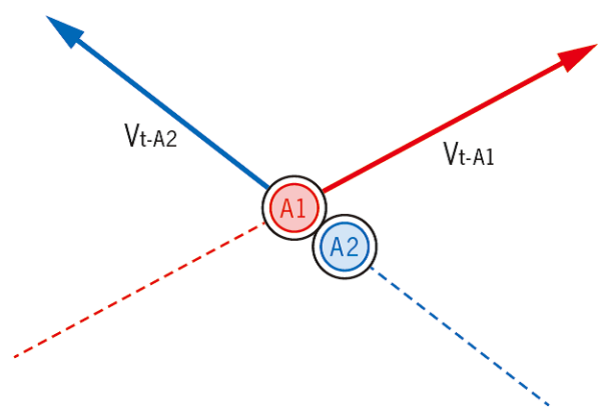

Fig. 13. AAs' original guidance to drive robots to the target positions: collision of robots.

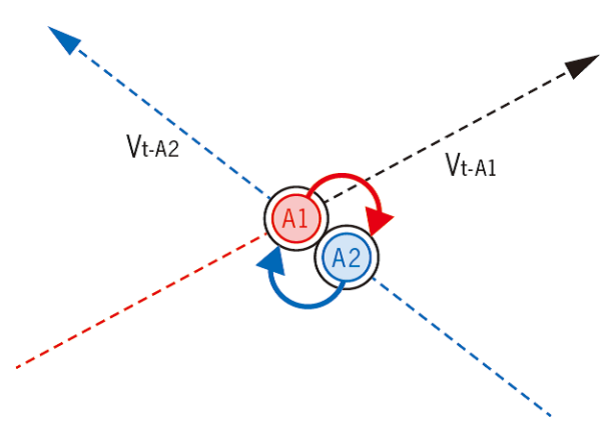

Fig. 14. AAs' original guidance to drive robots to the target positions: exchange of robots.

removes stagnation of convergence of formation in a simple way. The two robots can just keep moving as shown in Fig. 15. If a robot being driven by an AA collides with an unoccupied robot, the AA simply migrates to the unoccupied robot and keeps moving to the original target location.

In our system, each AA has its own coordinate system. Therefore, these coordinate systems must be integrated in the process of exchanging the robots. Each $\mathrm{PA}$ also has a vector value based on the coordinate system of the source AA, and each of them must be adjusted for the coordinate system of AA on which the PA

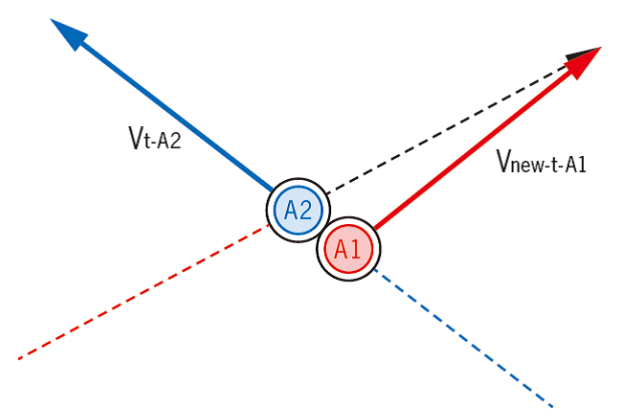

Fig. 15. AAs continue to move with new robots to the targets. 
arrives. If multiple PAs from different AAs arrive at the same AA, the PAs are synthesized into one PA. Let $n$ be the number of PAs that the AA receives, $\theta$ be the angle value of $\mathrm{AA}$ and $\theta_{i}$ be an angle of a PA that the AA receives. The angle of vector value that the integrated $\mathrm{PA}$ holds is updated as follows:

$$
\theta=\frac{1}{n+1}\left(\theta+\sum_{i=1}^{n} \theta_{i}\right) .
$$

The individual coordinates gradually converge and become stable as shown by Eq. (4).

\subsection{Pheromone agents' behavior}

In this subsection, we describe the algorithm for PAs (Algorithm 2). A PA is generated by an AA and contains information about the relative expected locations for the neighboring robots. The PA must find the proper AA and make it drive some robots to occupy those locations in order to compose the target formation. The PA diffuses the information through migrations while generating its clones among the robots in the field to find target AA to guide. First, the PA duplicates itself to make clones, and each of them migrates to the robots within the communication range of the current robot. A PA duplicates itself according to the number of robots in the communication range. Repeating this process, the PA ultimately reaches the target AA.

Figures 16 and 17 illustrate the process of the diffusion of PAs to other robots. As shown in Fig. 16, as soon as PAs are generated by their source AA, they migrate to all the robots in the communication range. As shown in Fig. 17, if there is not an AA on a robot to which the PA has migrated, the PA generates new clones, which further migrate to robots within the communication range. Because PAs repeatedly perform cloning in the diffusion process, PAs that are generated and dispatched from the

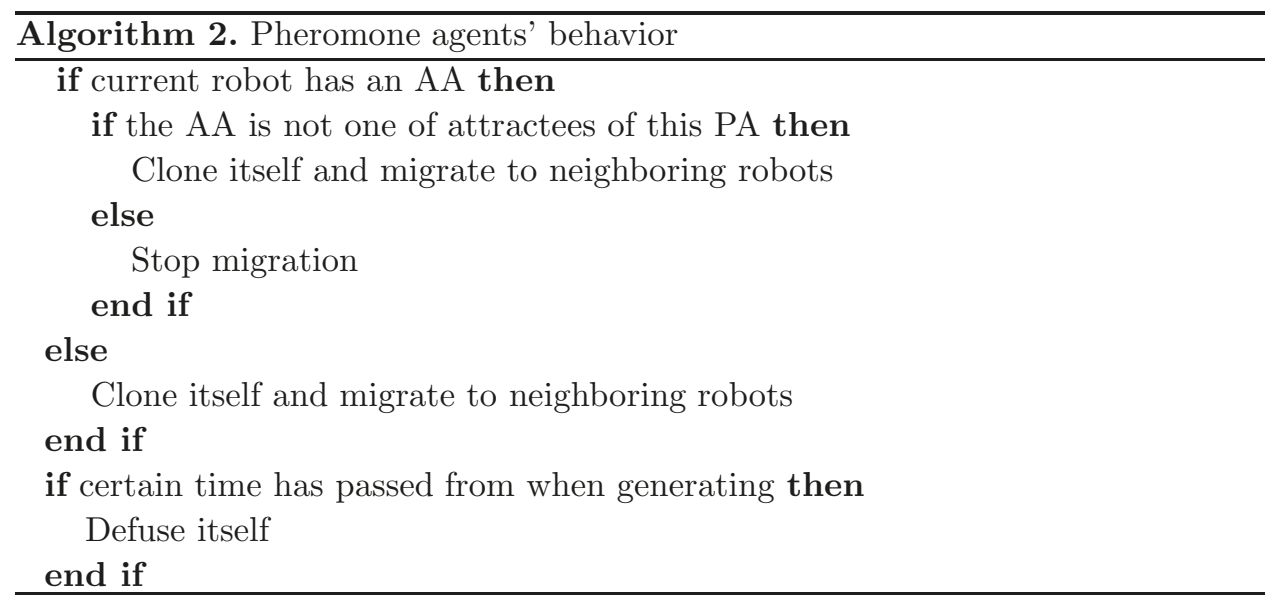




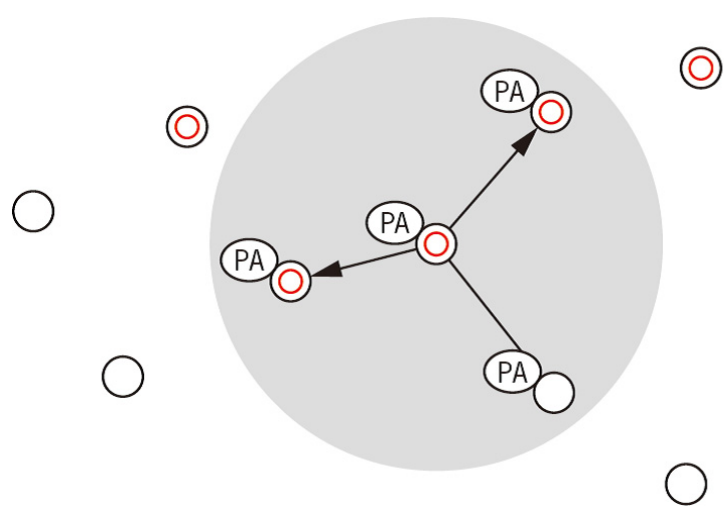

()

Fig. 16. (Color online) Cloned PAs move into neighboring robots. The red inner circle indicates that an $\mathrm{AA}$ is present.

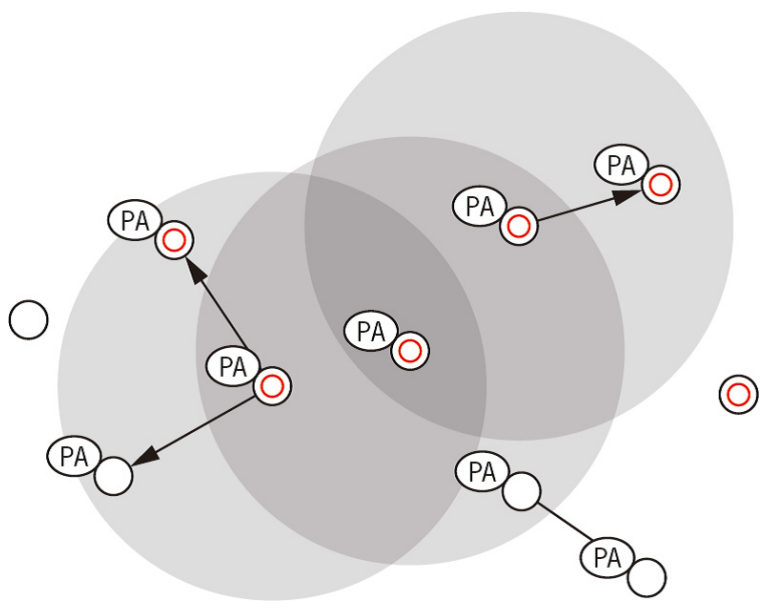

Fig. 17. PAs keep cloning to find the targets.

same source AA may reach a robot with an AA through different routes. In such a case, the target AA discards older PAs and uses the last PA as the guide using the source and target agent identifications and timestamp that are assigned to each PA when it is generated. PAs have duration time as the physical pheromone gradually evaporates, so that all the PAs gradually fade away as time elapses. Thus, the information conveyed by PAs is kept fresh. The items of the information that each PA has are as follows:

(1) ID of source ant agent,

(2) ID of target ant agent,

(3) the vector value pointing to the desired neighboring position of the source AA, i.e. the target position the other AA should drive a robot and make it occupy,

(4) the timestamp indicating when it was generated. 


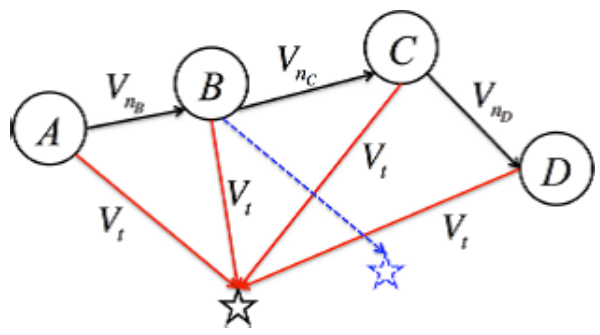

Fig. 18. PAs update a target vector when they migrate.

The vector values of PAs must always point to the desired neighboring position, even if the PAs repeatedly migrate to too many robots to find the target AA. Therefore, a vector value in a particular PA is updated to new vector value $V_{\text {new-target }}$ when the PA migrates from a robot to another robot. The $V_{\text {new-target }}$ is calculated using $V_{t}$ and a vector value $V_{\text {another }}$ from the current robot to another robot that is a destination of migration as follows:

$$
V_{\text {new-target }}=V_{t}-V_{\text {another }} \text {. }
$$

Figure 18 shows an example process where a PA updates $V_{t}$ along with the migration path $(A, B, C, D)$. Initially, the $\mathrm{PA}$ is on robot $A$ and $V_{t}$ points to the location where the neighboring robot should come to occupy as represented by the black star. Once it migrates to robot $B, V_{t}$ would point to the location represented by the blue star. In order to make $V_{t}$ keep pointing to the black star, the PA has to adjust $V_{t}$ through the calculation: $V_{t} \leftarrow V_{t}-V_{n B}$, where $V_{n B}$ is the vector to robot $B$ from robot $A$. In the same manner, the PA repeats adjustments of $V_{t}$ on robots $B, C$ and $D$ so that $V_{t}$ still points to the black star for robot $D$.

\section{Numerical Experiments}

In order to demonstrate the feasibility of our algorithm for formation control in three-dimensional environments, we have implemented our algorithm on a simulator and conducted numerical experiments. In the experiments, we have set the following experimental conditions.

(1) The experimental field is $1,000 \times 1,000 \times 1,000$ cubic space, and robots are randomly placed in the space.

(2) The range of communication network for each robot is 150 units and the range of the sensor is same as the above.

(3) Each robot can move 2 units in each time step.

(4) Interval time for generating PAs is 2 time steps and the duration time of each PA is 10 time steps.

(5) The initial locations and angles of robots are randomly determined without overlapping. 
(6) Each robot is represented as a circle on the grid space and emphasized as a red dot if it is being driven by an AA. A robot without an AA just sits still.

(7) Robots without AAs are depicted as blue circles.

We have conducted experiments for composing a solid cube formation. Figure 19 shows the images of the simulation. The images are snapshots of the process of composing a solid cube formation taken at every 100 steps. Figure 19(a) shows the state after 100 steps, Fig. 19(b) shows the state after 200 steps the Figs. 19(c) and $19(\mathrm{~d})$ show the states after 300 and 600 steps, respectively. As Figs. 19(a)-19(c)

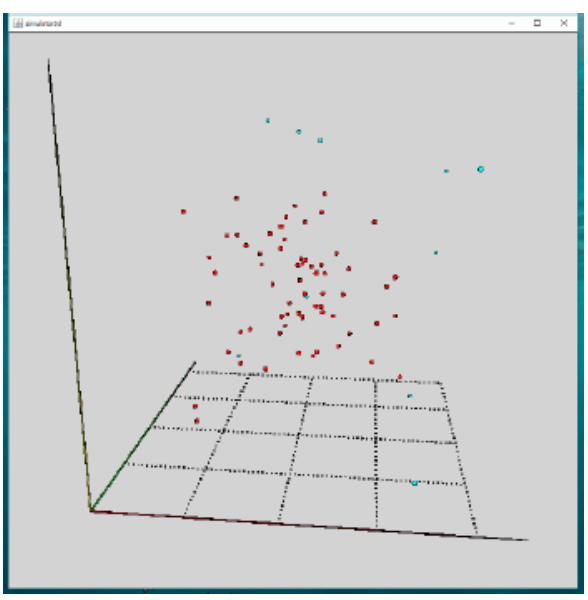

(a) 100 steps.

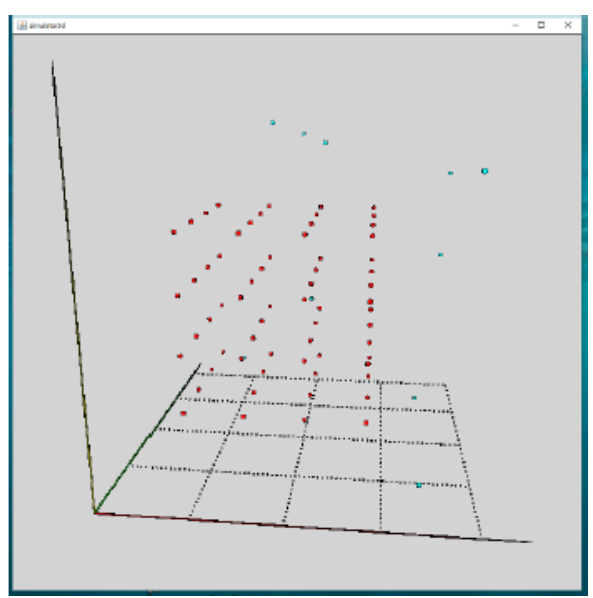

(c) 300 steps.

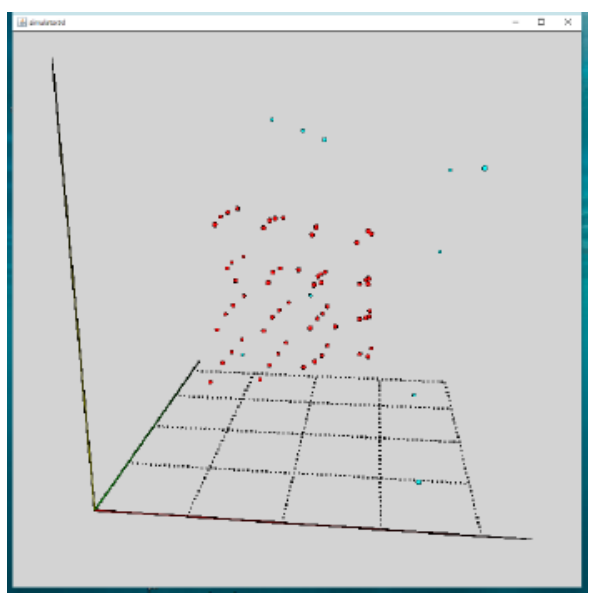

(b) 200 steps.

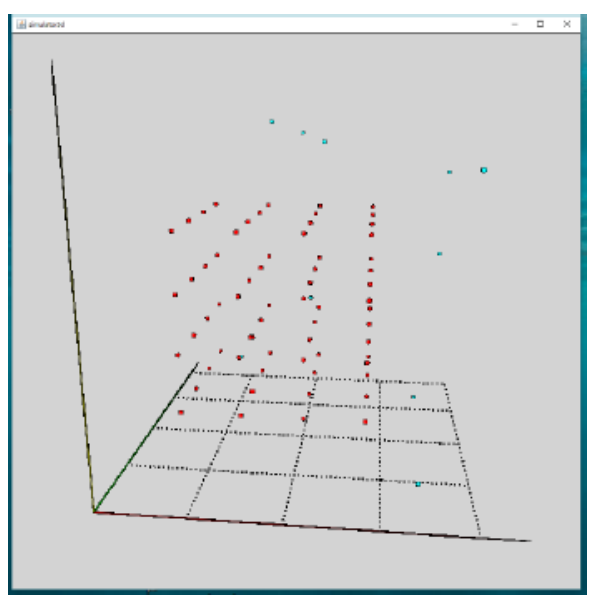

(d) 600 steps.

Fig. 19. Illustration of the emergence of a cubic formation. Panels (a)-(d) show the states after 100 steps, 200 steps, 300 steps and 600 steps, respectively. 
demonstrate, we can observe that the shape of a cube is gradually emerging as time progresses. When we compare Fig. 19(c) with Fig. 19(d), we can see that the shape at 300 steps is almost the same as the shape at 600 steps. Therefore, we can conclude that this formation converges at around 300 steps.

In order to investigate the behaviors of our formation control system in detail, we compared our formation with the following three approaches: (1) the simple approach where each robot has no volume and therefore, does not collide with each other; (2) non-exchange approach where each robot has a certain volume as ours, but AA does not exchange its driving robot with the other AA's driving robot upon collision; and (3) exchanging approach where each robot has a certain volume and the AA exchanges its driving robot with the other AA's driving robot upon collision.

First, we quantitatively compared how far each of the three approaches completes the desired target solid cubic formation. In the simulator, we can say that the formation task is completed when all the robots with AAs arrive at the target locations. In order to define the completion without ambiguity, we have set the allowable deviation from the ideal positions, and check whether the current locations of the robots are within the deviation in every time step. We have measured the differences between actual positions and the ideal positions, and calculated the average of them. In order to check the deviation, we have first calculated the barycenters of both actual and ideal positions, and then calculated all the coordinates of robots relative to the barycenters. During the simulation, we measure the distances between the relative coordinates of actual robots and the ideal relative coordinates, and have calculated the averages $D_{\text {average }}$ of the shape with the numbers of robots that are out of alignments as follows:

$$
D_{\text {average }}=\frac{1}{n} \sum_{i=1}^{n}\left\|I_{i}-A_{i}\right\| .
$$

In the equation, $I_{i}$ is the ideal relative coordinate of a robot with an AA from the barycenter, $A_{i}$ is the actual relative coordinate of the robot from the barycenter and $n$ is the number of AAs composing the formation. Figure 20 shows how the values of $D_{\text {average }}$ of the three approaches vary as time elapses. In the figure, the horizontal axis shows the time steps and the vertical axis shows the values of $D_{\text {average }}$ calculated by Eq. (6). In the experiment, the objective formation is a regular hexahedron, i.e. a cube, with a width of 500 units, the number of AAs to drive robots for composing the formation equal to 27 and the number of robots that are spread out in the field being 37, which is 10 more than the necessary number of robots. Therefore, AAs can pick up conveniently located 27 robots out of 37 robots.

As the line graphs of $D_{\text {average }}$ in the figure show, the simple approach without collision detection completed the formation at about 250 time steps. In the nonexchanging case, however, some robots collide with each other and cannot make progress. As the line graph of no migration, i.e. nonexchange approach, shows, $D_{\text {average }}$ stagnated at 50 units without converging to 0 units. Clearly this approach 


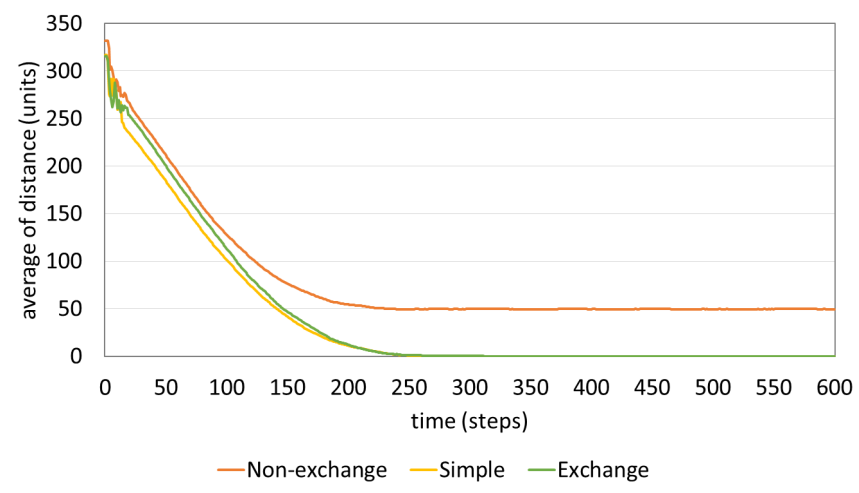

Fig. 20. Comparison of the values of $D_{\text {average }}$ for simple approach (ignoring clash judgement), the nonexchange approach (no migration) and the exchange approach (performing migration for exchanging robots).

makes the robots fail to compose the formation regardless of how long we continue the simulation, because several robots in the collisions prevent other robots from moving. On the other hand, in our exchange approach where the ant agents exchange the driving robots, $D_{\text {average }}$ becomes 0 units at about 260 steps. In addition, while the complete formation could not be achieved in the case of nonexchange approach, the exchange approach could complete the given formation. Furthermore, the figure shows that our exchanging approach has almost the same performance as the simple nonexchange approach. We believe that our approach where mobile agents exchange their driving robots is an essential method for formation completion for the realworld applications of multi-robot control.

Figure 21 shows the number of AAs that exchange their driving mobile robots in the formations with 8,27,64 and 125 robots, respectively. In the simulation, the objective formation is the same cubic shape as before, and the number of robots that are spread out in the field is 10 more than the necessary number of nodes. As shown in the figure, in the case of the small number of eight robots, AAs have scarcely

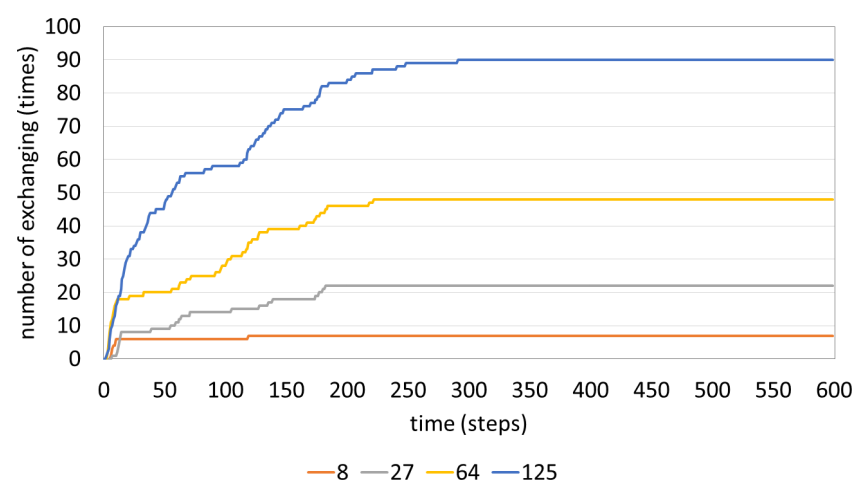

Fig. 21. Number of exchanging AAs in the formations with different numbers of robots. 
exchanged their driving robots; they have exchanged only seven times. In the shape with 27 robots, AAs exchanged 22 times, which is almost three times that of the case of eight robots. Furthermore, in the shape with 64 robots, AAs exchanged 48 times, and in the shape with 125 robots, AAs exchanged 90 times. Thus, we can say that the number of exchanging AAs increases more rapidly as the number of robots in the formation increases. The differences of the numbers of exchanges for shapes with 8 , 27, 64 and 125 robots also increase. That is, the number of exchanges exponentially increases as the number of robots for the given formation increases. We can presume the reason behind such phenomena as follows: the density in the formation increases as the number of robots in the formation increases. Since the width of the target shape is fixed, the more the density increases the more frequently collisions occur. We can conclude that the greater the number of robots that are participating, the more effective is our approach for the composition of a formation.

Figure 22 shows the values of $D_{\text {average }}$ for composing the formations with 8, 27, 64 and 125 robots. The formation is a solid cube as before. The simulation has taken 250 steps for composing a cubic formation with eight robots, while it has taken 350 steps for composing the same formation with 125 robots. It was much faster, i.e. 200 steps, in the shape composition with 27 robots. That is, it took $50 \%$ more completion time for 125 robots than for 27 robots, but the rate of increase is not as much as the number of exchanging AAs. When we investigate more closely the line graphs in the figure, we can observe that the average distances drastically decrease in the early stages of composing the formation. We can presume that the reason why we can observe such phenomena is our employment of mobile software agents. The distances of AAs to the target positions are reduced not through driving the mobile robots but through migrations of AAs. We can observe that the migrations of AAs for exchanging robots frequently occur in the very early stages of the simulation as can be seen in Fig. 21 and the steps around completing the formations as shown in Fig. 22. We can conclude from Figs. 21 and 22 that the time required to complete a formation does not increase as much as the number of exchanges of robots. This demonstrates

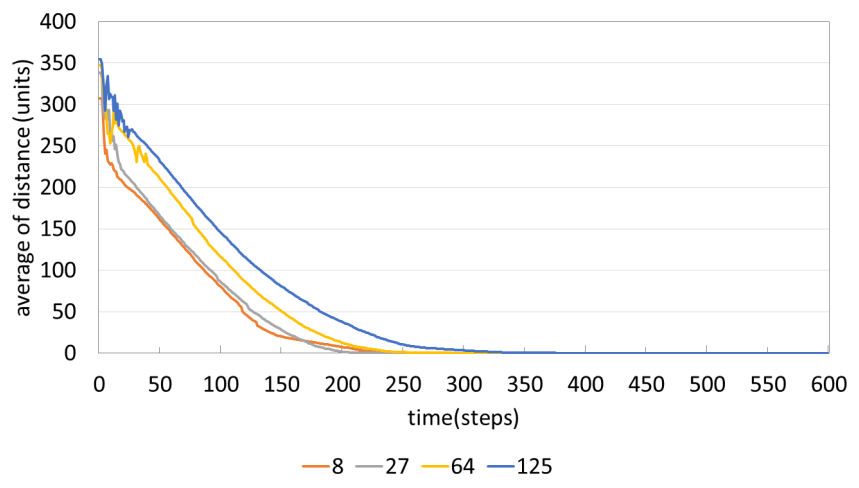

Fig. 22. Average of distances to the target positions in the formations with different numbers of robots: The cases of $8,27,64$ and 125 robots. 
the effectiveness of the mobile software agents, because software mobile agents can migrate instantly due to the attribute of software while physical robot maneuvers take much time. Exchanging robots is a simple migration of the mobile software agents.

As we have mentioned in Sec. 2, Mamei et al. and Stoy and Nagpal have proposed similar methods that compose formations of robots. ${ }^{41,43}$ Both of their multi-robot systems are self-configuring, similar to ours. The experiments of Mamei et al. are, however, entirely conducted on a two-dimensional surface, in contrast with our three-dimensional formation environment. The experiments of Stoy and Nagpal demonstrate the formation of more complex three-dimensional shapes than ours, such as an airplane-like shape. As they admit, however, communication in their system is time-consuming, and the implementation of motion is also far more timeconsuming; their system consumes a $2-100$-fold increase in time steps. The reasons are not only the challenging shape but also the load attributable simply to message exchange. Since our multi-robot system employs mobile agents to convey minimal but necessary information for movements to other robots, we have successfully reduced the communication overhead with our design.

\section{Conclusions}

We have proposed a formation control algorithm for swarm robots using mobile agents that avoids the computation costs of centrally directed control and computation and energy costs of collision avoidance and allows recovery from collision. Mobile agents drive physical robots utilizing indirect communication through pheromone diffusion. Pheromone diffusion is also achieved by mobile agents. In the algorithm, we have implemented two kinds of software mobile agents, namely ant agents and pheromone agents. Ant agents generate pheromone agents with local information. The local information consists of vector values that specify the locations that they want the neighboring robots to occupy in order to compose the objective formation. Pheromone agents, which are generated by an ant agent, migrate to other robots in order to attract other ant agents that drive robots and thus attract robots to compose the objective formation. The pheromone agents influence the ant agents and guide them to drive the physical robots to the proper locations. Once the pheromone agent arrives to an ant agent, it synthesizes the vector values that are given by other ant agents that have generated the pheromone agents with different vector values. Thus the pheromone agent that guides the ant agent has to produce a new vector value that points to the desired position. And then the ant agent starts driving the robot to the specified position. In addition, in the case where two robots with ant agents collide, they exchange their driving robots, and keep driving the exchanged robots to the original target positions assigned to the ant agents without any complex detour maneuvers. Eventually, all the robots occupy the most nearly correct positions and complete the composition of the target formation. 
In order to demonstrate the effectiveness of our algorithm, we have implemented a simulator and conducted numerical experiments. We have successfully demonstrated that our algorithm can make a fleet of mobile robots to compose a formation while improving the performance by exchanging the robots in case of collision even if the robots are densely packed in the target formation. We believe that our algorithm contributes an important concept to the multi-robot systems community.

As future work, we will be extending our method to compensate for the situation where some robots can no longer be controlled. In our algorithm, the mobile agents, which essentially play the roles of traditional robots, have no volume because they are just executable programs. They can move to their destinations without interfering with other robots. Driving mobile agents, i.e. the ant agents, can abandon their physically out-of-control robots and look for usable idle robots. This feature would contribute to the robustness of our swarm robot formation system. We are planning to construct a fleet of physical swarm robots to verify the feasibility of our enhanced algorithm. How to make stable formations in three-dimensional space would be one of the most challenging problems.

\section{Acknowledgments}

The authors appreciate Kimiko Gosney for her contribution in the completion of this paper. She gave us useful comments. This work is partially supported by the Japan Society for Promotion of Science (JSPS), with the basic research program (C) (Nos. 26350456, 26750076 and 17K01304), Grant-in-Aid for Scientific Research (KAKENHI) and Suzuki Foundation.

\section{References}

1. H. Yajima, R. Oikawa, M. Takimoto and Y. Kambayashi, Practical formation control of swarm robots using mobile agents, in Proc. SAI Intelligent Systems Conf. 2017 (ACM Press, 2017), pp. 1002-1009.

2. Y. Kambayashi and M. Takimoto, Higher-order mobile agents for controlling intelligent robots, Int. J. Intell. Inf. Technol. 1(2) (2005) 28-42.

3. W. Binder, J. G. Hulaas and A. Villazon, Portable resource control in the J-SEA12 mobile agent system, in Proc. 5th Int. Conf. Autonomous Agents (2001), pp. 222-223.

4. M. Takimoto, M. Mizuno, M. Kurio and Y. Kambayashi, Saving energy consumption of multi-robots using higher-order mobile agents, in Proc. 1st KES Int. Symp. Agent and Multi-Agent Systems, Lecture Notes in Computer Science, Vol. 4496 (Springer-Verlag, 2007), pp. 549-558.

5. J. Cheng, W. Cheng and R. Nagpal, Robust and self-repairing formation control for swarms of mobile agents, in Proc. 20th National Conf. Artificial Intelligence, Vol. 1 (AAAI Press, 2005), pp. 59-64.

6. M. Mizutani, M. Takimoto and Y. Kambayashi, Ant colony clustering using mobile agents as ants and pheromone, in Proc. 2nd Asian Conf. Intelligent Information and Database Systems Applications of Intelligent Systems, Lecture Notes in Computer Science, Vol. 5990 (Springer-Verlag, 2010), pp. 435-444. 
7. M. Shintani, S. Lee, M. Takimoto and Y. Kambayashi, Synthesizing pheromone agents for serialization in the distributed ant colony clustering, in Proc. Int. Conf. Evolutionary Computation Theory and Applications and Int. Conf. Fuzzy Computation Theory and Applications (SciTePress, 2011), pp. 220-226.

8. M. Shintani, S. Lee, M. Takimoto and Y. Kambayashi, A serialization algorithm for mobile robots using mobile agents with distributed ant colony clustering, in Proc. 15th Int. Conf. Knowledge-Based and Intelligent Information and Engineering Systems, Lecture Notes in Computer Science, Vol. 6881 (Springer-Verlag, 2011), pp. 260-270.

9. Nagata, M. Takimoto and Y. Kambayashi, Cooperatively searching objects based on mobile agents, Transactions on Computational Collective Intelligence XI, Lecture Notes in Computer Science, Vol. 8065 (Springer-Verlag, 2013), pp. 119-136.

10. M. Dorigo, M. Birattari and T. Stützle, Ant colony optimization-artificial ants as a computational intelligence technique, IEEE Computat. Intell. Mag. 1(4) (2006) 28-39.

11. M. Dorigo and L. M. Gambardella, Ant colony system: A cooperative learning approach to the traveling salesman, IEEE Trans. Evol. Comput. 1(1) (1996) 53-66.

12. S. Goss, S. Aron, J. L. Deneubourg and J. M. Pasteels, Self-organized shortcuts in the argentine ant, Naturwissenschaften 76 (1989) 579-581.

13. S. Goss, R. Beckers, J. L. Deneubourg, S. Aron and J. M. Pasteels, How trail laying and trail following can solve foraging problems for ant colonies, in Behavioural Mechanisms of Food Selection, NATO ASI series (Series G: Ecological Sciences), Vol. 20 (Springer, Berlin, 1990), pp. 661-678.

14. J. L. Deneubourg, S. Goss, N. R. Franks, A. B. Sendova-Franks, C. Detrain and L. Chreien, The dynamics of collective sorting: Robot-like ant and ant-like robot, in Proc. 1st Int. Conf. Simulation of Adaptive Behavior: From Animals to Animats (MIT Press, 1991), pp. 356-363.

15. T. Wang and H. Zhang, Collective sorting with multi-robot, in Proc. 1st IEEE Int. Conf. Robotics and Biomimetics (IEEE Press, 2004), pp. 716-720.

16. E. D. Lumer and B. Faiesta, Diversity and adaptation in populations of clustering ants, in Proc. 3rd Int. Conf. Simulation of Adaptive Behavior: From Animals to Animats 3 (MIT Press, 1994), pp. 501-508.

17. Y. Kambayashi, M. Ugajin, O. Sato, Y. Tsujimura, H. Yamachi, M. Takimoto and H. Yamamoto, Integrating ant colony clustering to a multi-robot system using mobile agents, Ind. Eng. Manage. Syst. 8(3) (2009) 181-193.

18. S. Sugiyama, H. Yamachi, M. Takimoto and Y. Kambayashi, Aggregating multiple robots with serialization, in Proc. 4th Asian Conf. Intelligent Information and Database Systems, Lecture Notes in Computer Science, Vol. 7196 (Springer-Verlag, 2012), pp. $177-186$.

19. K. Satta, M. Takimoto and Y. Kambayashi, Making autonomous robots form lines, in Proc. Fourth Asian Conf. Intelligent Information and Database Systems, Lecture Notes in Computer Science, Vol. 7196 (Springer-Verlag, 2012), pp. 198-207.

20. Y. Kambayashi, K. Satta, K. Shibata, S. Sugiyama, M. Takimoto and H. Yamachi, Clustering multiple robots with serialization, Int. J. Intell. Inf. Database Syst. 8(3) (2014) 224-243.

21. R. Oikawa, M. Takimoto and Y. Kambayashi, Distributed formation control for swarm robots using mobile agents, in Proc. 10th Jubilee IEEE Int. Symp. Applied Computational Intelligence and Informatics (2015), pp. 111-116.

22. R. Oikawa, M. Takimoto and Y. Kambayashi, Predictive distributed formation control for swarm robots using mobile agents, Trans. Automatic Control and Computer Science, 61(1) (2016) 83-90. 
23. R. Oikawa, M. Takimoto and Y. Kambayashi, Composing swarm robot formations based on their distributions using mobile agents, in EUMAS 2015, AT 2015: Multi-Agent Systems and Agreement Technologies, Lecture Notes in Computer Science, Vol. 9571 (Springer-Verlag, 2016), pp. 108-120.

24. M. Dorigo, SWARM-BOT: An experiment in swarm robotics, in Proc. 2005 IEEE Swarm Intelligence Symp. (IEEE Press, 2005), pp. 192-200.

25. F. Mondada, L. M. Gambardella, D. Floreano, S. Nolfi, J. L. Deneubourg and M. Dorigo, SWARM-BOTS: Physical interactions in collective robotics, IEEE Robot. Autom. Mag. 12 (2005) 21-28.

26. M. Dorigo, D. Floreano, L. M. Gambardella, F. Mondada, S. Nolfi, T. Baaboura, M. Birattari, M. Bonani, M. Brambilla, A. Brutschy, D. Burnier, A. Campo, A. L. Christensen, A. Decugnière, G. A. Di Caro, F. Ducatelle, E. Ferrante, A. Förster, J. Guzzi, V. Longchamp, S. Magnenat, J. Martinez Gonzalez, N. Mathews, M. A. Montes de Oca, R. O'Grady, C. Pinciroli, G. Pini, P. Rétornaz, J. Roberts, V. Sperati, T. Stirling, A. Stranieri, T. Stützle, V. Trianni, E. Tuci, A. E. Turgut and F. Vaussard, Swarmanoid: A novel concept for the study of heterogeneous robotic swarms, IEEE Robot. Autom. Mag. 20(4) (2012) 60-71.

27. R. Groß, M. Bonani, F. Mondada and M. Dorigo, Autonomous self-assembly in swarmbots, IEEE Trans. Robot. 22(6) (2006) 1115-1130.

28. F. Ducatelle, G. A. Di Caro, C. Pinciroli and L. M. Gambardella, Self-organized cooperation between robotic swarms, Swarm Intell. 5(2) (2011) 73-96.

29. R. Groß, E. Tuci, M. Dorigo, M. Bonani and F. Mondada, Object transport by modular robots that self-assemble, in Proc. 2006 IEEE Int. Conf. Robotics and Automation (IEEE Computer Society Press, 2006), pp. 2558-2564.

30. R. O'Grady, C. Pinciroli, R. Groß, A. L. Christensen, F. Mondada, M. Bonani and M. Dorigo, Swarm-bots to the rescue, in Proc. European Conf. Artificial Life, eds. G. Kampis, I. Karsai and E. Szathmary, Lecture Notes in Computer Science, Vol. 5777 (Springer-Verlag, 2011), pp. 165-172.

31. V. Trianni, S. Nolfi and M. Dorigo, Cooperative hole avoidance in a swarm-bot, Robot. Auton. Syst. 54(2) (2006) 97-103.

32. E. Tuci, C. Ampatzis and M. Dorigo, Evolving neural mechanisms for an iterated discrimination task: A robot based model, in Proc. 8th European Conf. Artificial Life, Lecture Notes in Computer Science, Vol. 3630 (Springer-Verlag, 2005), pp. 231-240.

33. R. Luna and K. E. Bekris, Push and swap: Fast cooperative pathfinding with completeness guarantees, in Proc. 21nd Int. Joint Conf. Artificial Intelligence (2011), pp. 294-300.

34. J. Yu and S. M. LaValle, Planning optimal paths for multiple robots on graphs, in Proc. IEEE Int. Conf. Robotics and Automation (IEEE Computer Society Press, 2013), pp. $3612-3617$.

35. M. A. Erdmann and T. Lozano-Pérez, On multiple moving objects, in Proc. IEEE Int. Conf. Robotics and Automation (IEEE Computer Society Press, 1986), pp. 1419-1424.

36. P. Surynek, An optimization variant of multi-robot path planning is intractable in Proc. 24th AAAI Conf. Artificial Intelligence (AAAI Press, 2010), pp. 1261-1263.

37. T. Balch and R. C. Arkin, Behavior-based formation control for multirobot teams, IEEE Trans. Robot. Autom. 14(6) (1998) 926-939.

38. M. A. Lewis and K. Tan, High precision formation control of mobile robots using virtual structures, Auton. Robots 4(4) (1997) 387-403.

39. A. K. Das, R. Fierro, V. Kumar, J. P. Ostrowski, J. Spletzer and C. J. Taylor, A visionbased formation control framework, IEEE Trans. Robot. Autom. 18(5) (2002) 813-825. 
40. C. Unsal and J. S. Bay, Spatial self-organization in large populations of mobile robots, in Proc. 9th IEEE Int. Symp. Intelligent Control (IEEE Computer Society Press, 1994), pp. 249-254.

41. M. Mamei, M. Vasirani and F. Zambonelli, Experiments of morphogenesis in swarms of simple mobile robots, Appl. Artif. Intell. 18(9-10) (2004) 903-919.

42. A. Kondacs, Biologically-inspired self-assembly of two-dimensional shapes using global-to-local compilation, in Proc. 18th Int. Joint Conf. Artificial Intelligence (2003), pp. 633-638.

43. K. Sty and R. Nagpal, Self-reconfiguration using directed growth, in Proc. 7th Int. Symp. Distributed Autonomous Robotic Systems (2004), pp. 1-10.

44. N. Gordon, I. A. Wagner and A. M. Bruckstein, Discrete bee dance algorithm for pattern formation on a grid, in Proc. 2003 IEEE/WIC Int. Conf. Intelligent Agent Technology (2003), pp. 545-549.

45. M. Rubenstein and W. M. Shen, A scalable and distributed approach for self-assembly and self-healing of a differentiated shape, in Proc. 2008 IEEE/RSJ Int. Conf. Intelligent Robots and Systems (2008), pp. 1397-1402. 